\title{
THERMAL TESTING ON PROGRAMMABLE LOGIC DEVICES
}

\author{
Sergio Lopez-Buedo, Javier Garrido, and Eduardo Boemo \\ Laboratorio de Microelectrónica, Escuela Técnica Superior de Informática, \\ Universidad Autónoma de Madrid, Ctra. de Colmenar Km.15, 28049 Madrid, Spain. \\ e-mail: \{ sergio.lopez-buedo, javier.garrido, eduardo.boemo\}@ii.uam.es
}

\begin{abstract}
In this work, an FPGA-oriented temperature monitoring scheme is presented. A control circuit enables a ring-oscillator during a short period and measures its output frequency, a magnitude that is a function of the die temperature. Several sensors have been constructed using Xilinx chips, obtaining sensitivities between $17 \mathrm{kHz}$ per ${ }^{\circ} \mathrm{C}$ and $77 \mathrm{kHz}$ per ${ }^{\circ} \mathrm{C}$. The characterization of self-heating, matching between identical sensors, power supply dependence and detection of signal contentions has also been performed. The usefulness of other chip resources as thermal transducers, like the built-in OSC4 cell or the IOB clamping diodes, has also been verified. The use of ringoscillators convert the FPGAs in a powerful tool for researchers interested in thermal modeling of integrated circuits. Just the possibility of "moving" a sensor (or an array of them) over the die, in a simple, fast, and inexpensive way, is almost impossible in any other VLSI technology.
\end{abstract}

\section{INTRODUCTION}

The speed, gate density, and small packages of current fieldprogrammable logic devices (FPGAs) have appended thermal considerations to the traditional design trade-offs. The consumption associated to a given configuration is unknown a priori; thus, the particular features of a circuit (fine-grain pipelines, high-activity heavily loaded buses, etc.) can produce an unforeseen power overhead. In addition, some designer's errors like signal contentions, inadequate timing of the RAM modules [1], or a wrong implementation of the configuration scheme also increase chip temperature. Consequently, the inclusion of a thermal monitoring unit allows the user to detect several problems. However, in large FPGA-based systems is difficult to employ classic thermal transducers [2]: thermocouples, thermistors, or integrated sensors require both extra wiring and circuitry that must be immune to the influence of the high-frequency signals usually present on the board. Moreover, the designer must pay attention to topics beyond the scope of fast-prototyping, like sensor positioning, thermal coupling, or analog instrumentation.

The implementation of on-chip sensors is an alternative to avoid several of the inconveniences described above. However, the main available techniques [3], [4] require a full-custom chip redesign; thus they appear inadequate to end-users of commercial chips. An ingenious alternative to overcome this limitation was proposed by Peter Alfke [5]: to calculate the die temperature by measuring the junction forward voltage of the clamping diodes located in the FPGA pads. This technique lets the designer to obtain free, small and abundant on-chip thermal sensors at the cost of some external analog circuitry.
In this paper, a second alternative is proposed: by employing ring oscillators as temperature transducer [6], a type of circuit that can be easily implemented with few FPGA elements. The advantages of this approach are multiple: a) like other on-chip sensors, the junction temperature instead of the package one is measured; b) even considering that temperature is an analog magnitude, all signals result digital; thus, they can be processed using the general resources of the board; c) the sensor itself is small: practical circuits use two logic blocks, and a minimumsize sensor can be fitted in just an I/O block; d) a sensor or even an array of them can be placed in any position of the chip, making possible the construction of a thermal map of the die; and $\mathrm{f}$ ) the sensor can be dynamically inserted or eliminated.

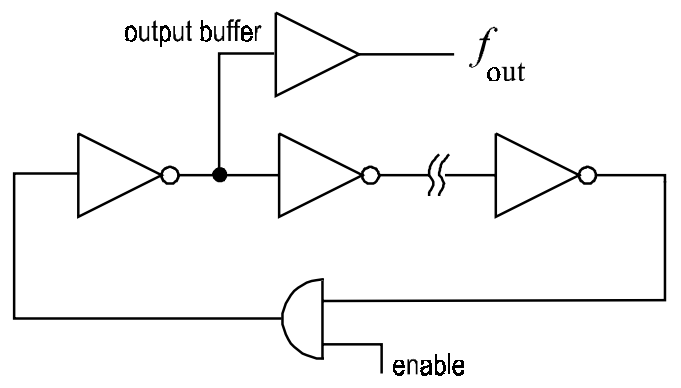

Fig. 1: A ring-oscillator scheme

This paper is organized as follows. In Section 2, the main characteristics of the sensors are presented. Then, the setup of the experiments is described. In Section 4, the response of each circuit is detailed and their usefulness for on-line testing is verified. Finally, the main conclusions are summarized.

\section{ON-CHIP SENSORS FOR FPGAs}

Ring-oscillators (Fig.1) can be mapped on FPGAs using the look-up tables (LUTs) or the programmable inverters included in the input-output block (IOBs). An odd number of inversions produces the necessary phase shifting to start the oscillation. The resulting period is twice the sum of the delays of all the elements that compose the loop. In practical applications, it is useful to insert an external signal to disable the oscillator, and an output buffer to prevent frequency variations.

This paper is mainly focused on XC4000 series chips and CLBbased oscillators, but preparatory studies including XC3000 circuits as well as IOB-based rings can be found in [7]. From all 
possible ring implementations, three classes of circuits, called osc1, osc2 and osc3, have been characterized. In addition, four identical oscillators (osc5 to osc8) situated in the chip corners have been constructed in order to determine the degree of sensor matching, as well as to detect thermal gradients. Finally, the thermal response of the $8-\mathrm{MHz}$ output of the built-in clock generator osc4 cell [8] has also been measured. Main circuit features are summarized in Tables 1 and 2.

\begin{tabular}{|c|c|}
\hline Circuit & Features \\
\hline osc1 & $\begin{array}{l}\text { high net delay, } 2 \text { CLBs, } \\
\text { four F/G LUTs plus two H LUTs }\end{array}$ \\
\hline osc 2 & $\begin{array}{l}\text { similar net and LUT delays, } 2 \text { CLBs, } \\
\text { four F/G LUTs plus two H LUTs }\end{array}$ \\
\hline osc 3 & $\begin{array}{l}\text { high LUT delay, } 2 \times 2 \text { CLBs, } \\
\text { eight F/G LUTs plus four H LUTs }\end{array}$ \\
\hline $\operatorname{osc} 4$ & $8 \mathrm{MHz}$ built-in oscillator cell. \\
\hline osc 5 to osc 8 & $\begin{array}{l}\text { high LUT delay, } 2 \times 2 \text { CLBs, situated in } \\
\text { the die corners. }\end{array}$ \\
\hline
\end{tabular}

Table 1: Main characteristics of the oscillators.

\begin{tabular}{|l|l|l|}
\hline Circuit & LUT delays [ns] & net delays [ns] \\
\hline osc1 & 12.6 & 26.0 \\
\hline osc2 & 12.6 & 11.6 \\
\hline osc3 & 25.2 & 11.9 \\
\hline osc5 to osc8 & 23.2 & 11.8 \\
\hline
\end{tabular}

Table 2: Ring-oscillator loop delay components (Xilinx Timing Analyzer data).

For the sake of completeness, Alfke's solution has also been implemented. This method is based on the voltage-current relation of an ideal diode, depicted in Eq.1:

$$
I=I_{s}\left(e^{q V / k T}-1\right)
$$

where $I_{s}$ includes six parameters that depend on the temperature, which practically determine the overall thermal characteristics of the junction. In short, for a fixed voltage, the current rises almost exponentially with $\mathrm{T}$, meanwhile for a fixed current, the voltage across the junction diminishes almost linearly with T [9]. Practical applications [10], [11] have used the second operation mode. In Fig.2, a simplified structure of an IOB is depicted along with the measurement scheme utilized in this paper. The output buffer was tri-stated, meanwhile a current sink circuit was used to fix $1 \mathrm{~mA}$ in the lower diode.

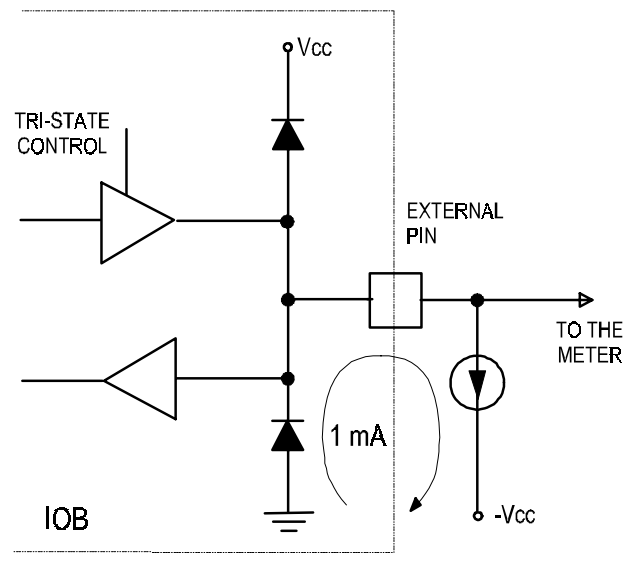

Fig. 2: IOB diode as thermal sensor.

\section{EXPERIMENT SETUP}

Sensors calibration was done by introducing the chip sample in a temperature-controlled oven. An Iron-Constantan (Fe-CuNi) thermocouple was placed in the center of the package, and was fixed to it with a heat conductive silver epoxy (a study about mechanical details of thermal sensors can be found in [8]). The error in the case temperature estimation was maintained near 1 ${ }^{\circ} \mathrm{C}$. In the oscillator-based sensors, long ribbon cables (near 0.8 meters) were utilized to carry the frequency outputs outside the oven. A driver $74 \mathrm{HC} 125$ was inserted to isolate the FPGA from these cables, in order to prevent excessive sensor power consumption due to these high off-chip loads.

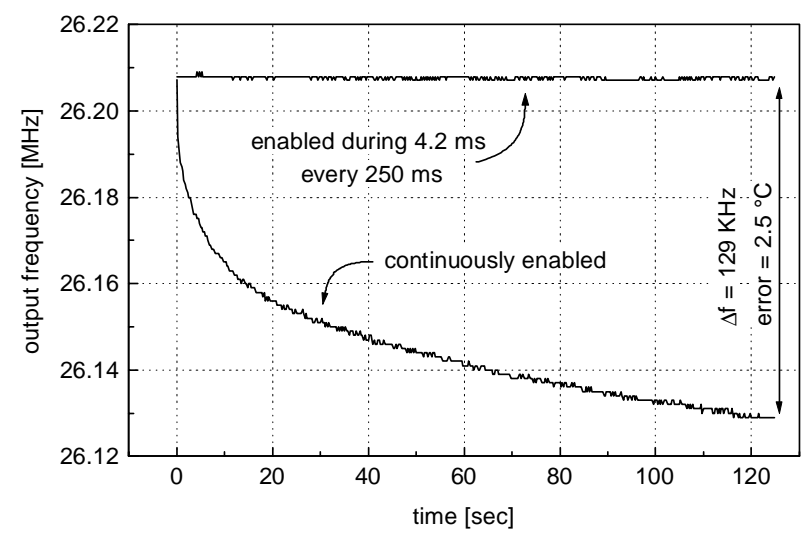

Fig. 3: Error in osc5 calibration. Short enable versus continuous operation. Sample rate: $250 \mathrm{~ms}$.

A custom instrumentation based on a $68 \mathrm{HC} 11$ microcontroller and an XC3130 FPGA was designed to count the frequencies. It allowed us to reduce the error caused by the own sensor dissipation. Each measurement was performed in two steps using a short enable window of $4.2 \mathrm{~ms}$. First, the oscillator was left running during $0.2 \mathrm{~ms}$ to stabilize the output. Then, the frequency measurement was performed during the following 4 
ms. Although this strategy improves the sensor characterization, in a practical application it can be avoided, considering that the error caused by self-heating was found less than $3{ }^{\circ} \mathrm{C}$. This fact is illustrated in Fig.3. The output frequency of osc5 was captured every $250 \mathrm{~ms}$, using both short enable and free-running operation. In the last case, a frequency drift of more than 129 $\mathrm{KHz}$ (equivalent to $2.5^{\circ} \mathrm{C}$ ) can be observed. This effect is negligible in the short enable operation mode.

\section{RESULTS}

In Figs. 4 and 5, the main experimental results are shown. All sensors exhibit a linear temperature dependence in the normal range of operation. The outputs of rings with identical layout (Fig.5) can differ as much as $3.6 \%$. As a consequence, any array of sensors to study the die temperature must be individually calibrated. However, their frequency variations respect to the room temperature values are similar. The power supply dependence of all oscillators result linear in the operation range, as is depicted in Fig. 6. Sensors whose loop delay is mainly caused by wiring are slightly less sensitive to power supply fluctuations.

In Fig.7, the results of the diode-based experiments are summarized. Two sensors, D1 and D2, have been characterized using different chip models, an XC4005EPC84 and an $\mathrm{XC} 3030 \mathrm{PC} 84-125$ respectively. In this case, a linear fit around $80^{\circ} \mathrm{C}$ shows slopes of $-1.2 \mathrm{mV}$ per ${ }^{\circ} \mathrm{C}$ and $-1.4 \mathrm{mV}$ per ${ }^{\circ} \mathrm{C}$. Diodes as thermal sensors result less linear than oscillators; however, they exhibit less power-supply dependence. For example, a voltage variation of $1 \%$ have been measured in the D1 sensor, for Vcc ranging between 4.5 and 5.5 volts.

In Fig.8, two examples of thermal testing are summarized. In the top graph, an intentional short-circuit between pins 35 and 36 (fixed to opposite logic levels) was produced. Both pins are situated in the bottom left corner, close to osc6. The graph shows the normalized frequency of the sensors (respect to their values at room temperature). Each output was sampled every $250 \mathrm{~ms}$. In $\mathrm{t}=0$, the short-circuit begins, finishing at $\mathrm{t}=25 \mathrm{sec}$. A data analysis indicates that all frequencies decrease following a second-order exponential; however, the decay is different for each sensor, pointing a temperature gradient. A peak temperature was detected by osc6: near $3^{\circ} \mathrm{C}$ higher than the measured in the opposite corner. Temperature in the equidistant corners (osc5 and osc7) resulted almost equivalent. In the bottom graph, the experiment was repeated for a short-circuit produced in two internal tri-state buffers situated near osc6. Even considering the small magnitudes involved in this case, the behavior is similar to the obtained in the previous test.

\section{CONCLUSIONS}

A group of experiments demonstrating the feasibility of on-line thermal monitoring on FPGAs have been presented. The die temperature have been exactly measured, without using the junction-to-case thermal resistance parameter, a value that can vary from one application to another. The designer can automatically detect several type of failures with the addition of a simple digital circuitry.

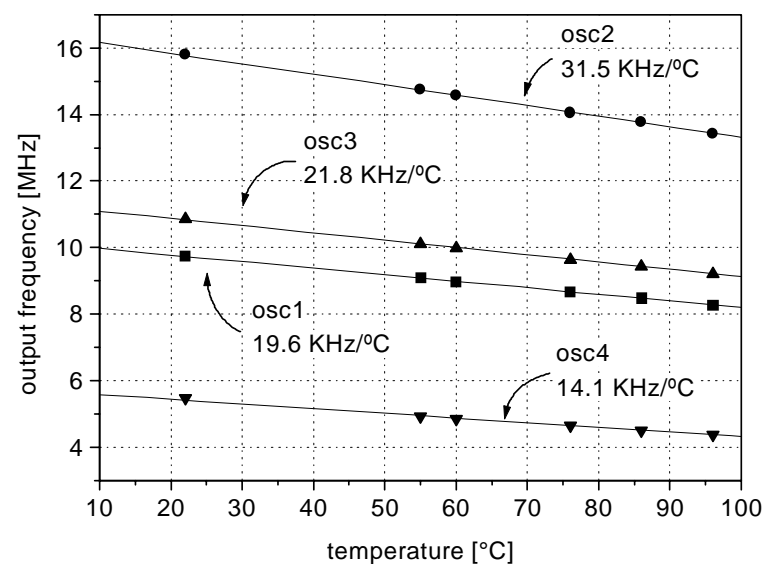

Fig. 4: Output frequency vs. temperature, osc1 to osc4.

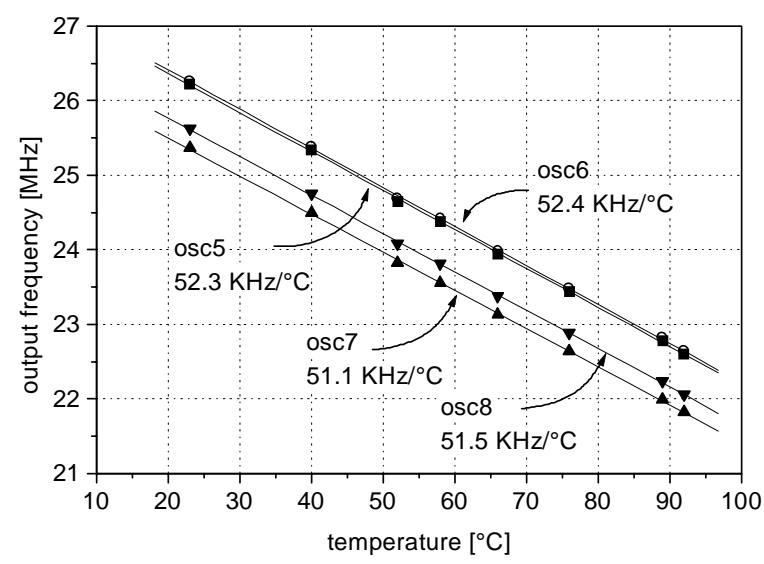

Fig. 5: Output frequency vs. temperature, identicallayout sensors osc 5 to $\operatorname{osc} 8$.

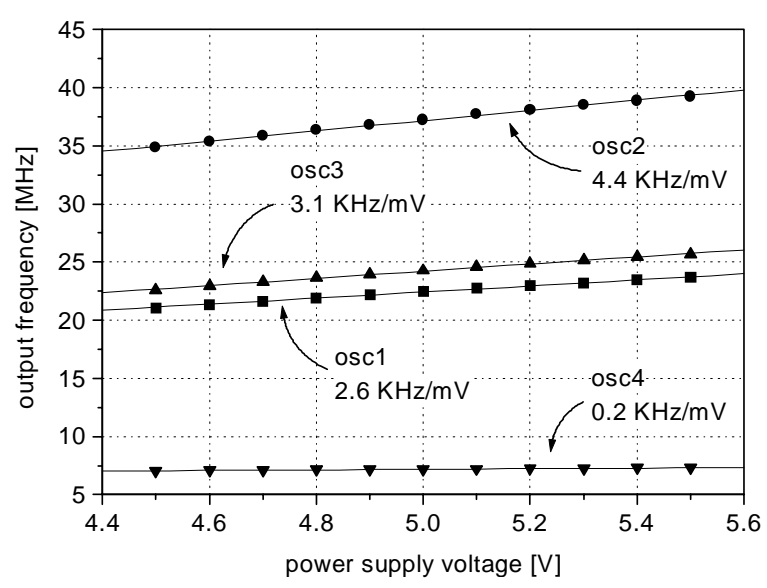

Fig.6: Output frequency vs. Temperature, 4005EPC84-3 


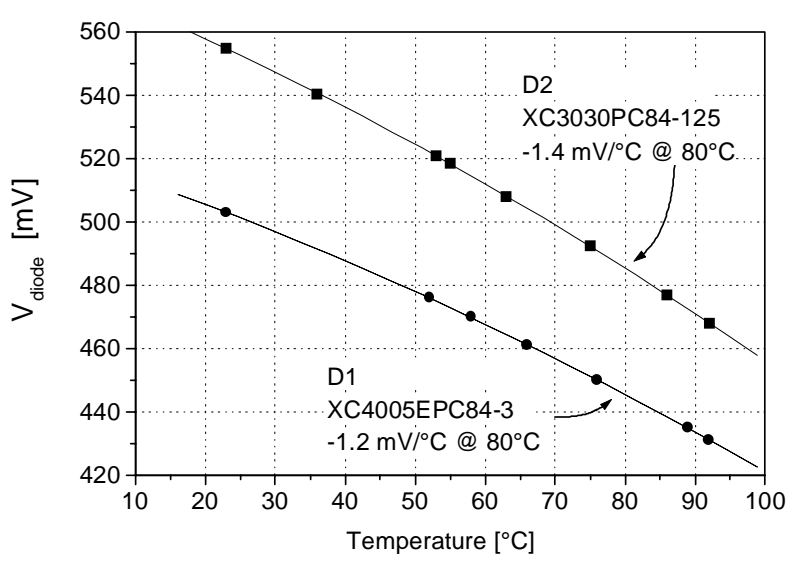

Fig. 7: Diode junction voltage vs. temperature.
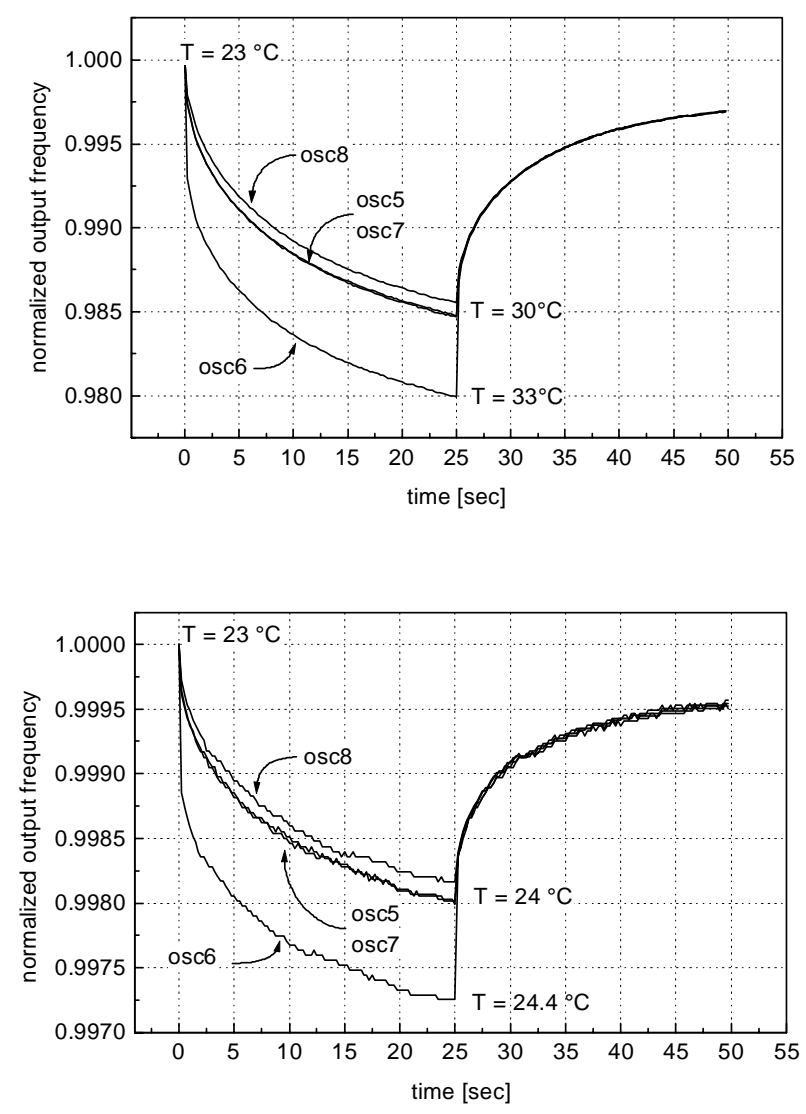

Fig.8: Thermal testing of signal contention using ringoscillators. Top: short-circuit at output pins. Bottom: short-circuit at internal long-line buffers.
All prototypes show a linear response in relation to temperature, but the best results in frequency range, resource occupation, and power supply sensitivity corresponded to the built-in XC4000series oscillator. However, the main disadvantage of this circuit is its fixed position in a corner of the chip. On the contrary, CLB-based ring oscillators can be situated in virtually any position.

This paper also suggests a new field of application for FPGAs. The reconfiguration capability transform these devices in a powerful tool for the study of thermal aspects of integrated circuits, allowing the researchers to perform an unlimited number of experiments.

\section{ACKNOWLEDGES}

This work has been supported by the Spanish CICYT under contract TIC95-0159. The authors wish to thank Xilinx Inc. for the donation of the tools.

\section{REFERENCES}

[1] Xilinx Inc., "Single-Port Edge-Triggered Mode", in The Programmable Logic Databook, pp.4-16, 1996.

[2] F. Goodenough, "Monolithic Silicon Temperature Sensors Challenge Thermistors, RTDs, and Thermocouples", Electronic Design, pp.54-64, October 1997.

[3] Wolffenbuttel, R., (ed.), "Silicon Sensors and Circuits. Onchip compatibility”, London: Chapman \& Hall, 1996.

[4] V. Székely, Cs. Márta, Z. Kohari and M. Rencz, "CMOS sensors for On-Line Thermal Monitoring of VLSI Circuits", IEEE Trans. on VLSI Systems, pp.270-276, Sep.1997.

[5] P. Alfke, Contribution to the comp.arch.fpga newsgroup, April 1997.

[6] G. Quenot, N. Paris and B. Zavidovique, "A temperature and voltage measurement cell for VLSI circuits", Proc. 1991 EURO ASIC Conf., pp-334-338, IEEE Press, 1991.

[7] E. Boemo and S. Lopez-Buedo, "Thermal Monitoring on FPGAs using Ring-Oscillators", Lecture Notes in Computer Science (Special Issue on FPL'97 Workshop), No 1304, pp.6978, Berlin: Springer-Verlag, 1997.

[8] Xilinx Inc., “Xact Libraries Guide”, pp. 3-388, April 1994.

[9] P. Gray, D. DeWitt, A. Boothroyd, and J. Gibbons, "Physical Electronics and Circuit Models of Transistors", John Wiley \& Son Inc., New York: 1964.

[10] L. Mahalingam, J. Adrews, and J. Drye, "Thermal Studies on PGA Packages for High Density LSI and VLSI Logic Circuits", IEEE Trans. on Components, Hybrids and Manufacturing Technology, Vol.CHMT-6, №3, pp.246-256, Sept. 1983.

[11] Xilinx Inc., "Packages Thermal Characterization Methods \& Conditions", in The Programmable Logic Databook, pp.10-6, 1996. 